\title{
Managing Dyslipidemia in Chronic Kidney Disease
}

\author{
Daniel E. Weiner, MD, Mark J. Sarnak, MD
}

\begin{abstract}
OBJECTIVE: Patients with chronic kidney disease (CKD) are at higher risk for cardiovascular disease (CVD) than patients in the general population. One potentially modifiable risk factor for CVD in patients with CKD is dyslipidemia. In the current manuscript we review observational and trial data assessing dyslipidemia and its treatment in this patient population.
\end{abstract}

RESULTS: Observational studies have noted a "reverse epidemiology" in patients with CKD such that low total cholesterol levels are associated with a higher mortality rate. The complex lipid profile of patients with CKD also raises questions as to whether lipid-lowering therapy will be beneficial in this patient population. Although there are only a few trials assessing the relationship between lipid-lowering therapy and CVD outcomes in CKD patients, many lipid-lowering medications are both safe and effective. In addition, there is suggestive evidence that statin therapy, in particular, also may reduce inflammation and slow the decline in glomerular filtration rate (GFR) in patients during the earlier stages of CKD.

CONCLUSION: Because of the high rate of CVD in patients with CKD and the overall safety of most medical therapies for dyslipidemia in CKD, current guidelines from the National Kidney Foundation Kidney Disease Outcomes Quality Initiative recommend aggressive therapy of dyslipidemia. These guidelines do, however, acknowledge the paucity of trial data in this patient population. There are 3 ongoing randomized controlled trials that are assessing the effect of statin therapy on CVD outcomes. These studies will hopefully provide definitive answers as to the appropriate treatment of dyslipidemia in CKD.

KEY WORDS: cardiovascular disease; dyslipidemia; hydroxymethylglutaryl-CoA reductase inhibitors; chronic kidney disease; dialysis.

J GEN INTERN MED 2004;19:1045-1052.

$\mathrm{C}$ hronic kidney disease (CKD) is a growing health burden in the United States, with estimates of nearly 20 million affected. ${ }^{1}$ CKD is defined as either a sustained reduction in kidney function with a glomerular filtration

Received from Division of Nephrology (DEW, MJS), Tufts-New England Medical Center, Boston, Mass.

Address correspondence and requests for reprints to Dr. Sarnak: Box 391, Tufts-New England Medical Center, 750 Washington Street, Boston, MA 02111 (e-mail: msarnak@tufts-nemc.org). rate (GFR) of less than $60 \mathrm{ml} / \mathrm{min} / 1.73 \mathrm{~m}^{2}$ or evidence of kidney damage. Although kidney damage may be defined by radiologic or pathologic findings, it is diagnosed most frequently by the presence of microalbuminuria. Levels of urinary albumin excretion above $30 \mathrm{mg} /$ day (or urinary albumin to creatinine ratios of $17 \mathrm{mg} / \mathrm{g}$ or higher for men or $25 \mathrm{mg} / \mathrm{g}$ or higher for women) on at least 2 measurements are consistent with CKD, regardless of the level of GFR. In the current review, basing our definitions of stages of CKD on the National Kidney Foundation (NKF) Kidney Disease Outcomes Quality Initiative (K/DOQI) guidelines, ${ }^{1}$ we will focus primarily on the epidemiology of dyslipidemia and its therapy in patients with Stage $5 \mathrm{CKD}$ (defined as dialysis or GFR $<15 \mathrm{ml} / \mathrm{min} / 1.73 \mathrm{~m}^{2}$ ), but also discuss studies in patients with Stage 3 and 4 CKD (GFR from 15 to $59 \mathrm{ml} / \mathrm{min} / 1.73 \mathrm{~m}^{2}$ ) and kidney transplant recipients.

Patients with CKD are at high risk for cardiovascular disease (CVD), ${ }^{2,3}$ and an increased prevalence of both CVD morbidity and mortality is evident at all ages among patients with CKD. ${ }^{4}$ Both traditional risk factors, including diabetes, dyslipidemia, and hypertension, and nontraditional risk factors associated with $\mathrm{CKD}$, including inflammation, oxidant stress, malnutrition, and proteinuria, may further increase CVD risk. ${ }^{5,6}$

Chronic kidney disease itself, whether manifested by reduced GFR or microalbuminuria, is an independent risk factor for CVD outcomes. ${ }^{7}$ There are several potential explanations for this finding but one possibility is that CKD may represent the kidney manifestation of the systemic burden of vascular and endothelial disease. ${ }^{8,9}$ Another possibility is that CKD reflects the severity and duration of traditional CVD risk factors such as hypertension, diabetes, and dyslipidemia. ${ }^{10}$

National guidelines have identified dyslipidemia, and elevated levels of low-density lipoprotein cholesterol (LDL-C) in particular, as a key risk factor for CVD risk modification in the general population. ${ }^{5}$ Many patients are unable to achieve lipid goals established in the clinical guidelines through lifestyle changes alone, ${ }^{11}$ and, for these patients, guidelines advise pharmacologic therapy. Based on the risk reductions achieved in the general population in patients with or at high risk for CVD, ${ }^{12-15}$ the small amount of existing data in the CKD population, and the marked risk of CVD in patients with kidney disease, the NKF K/DOQI has established guidelines for dyslipidemia therapy in $\mathrm{CKD} .^{3}$ 
Table 1. Lipid Abnormalities by Target Population (Approximate Percentage)

\begin{tabular}{|c|c|c|c|c|}
\hline & $\begin{array}{c}\text { Total Cholesterol } \\
>240 \mathrm{mg} / \mathrm{dl}\end{array}$ & $\begin{array}{l}\text { LDL Cholesterol } \\
>130 \mathrm{mg} / \mathrm{dl}\end{array}$ & $\begin{array}{l}\text { HDL Cholesterol } \\
<35 \mathrm{mg} / \mathrm{dl}\end{array}$ & $\begin{array}{c}\text { Triglycerides } \\
>200 \mathrm{mg} / \mathrm{dl}\end{array}$ \\
\hline General population* & 20 & 40 & 15 & 15 \\
\hline $\begin{array}{l}\text { CKD Stages } 1 \text { to } 4^{\dagger} \\
\text { With nephrotic syndrome } \\
\text { Without nephrotic syndrome }\end{array}$ & $\begin{array}{l}90 \\
30\end{array}$ & $\begin{array}{l}85 \\
10\end{array}$ & $\begin{array}{l}50 \\
35\end{array}$ & $\begin{array}{l}60 \\
40\end{array}$ \\
\hline $\begin{array}{l}\text { CKD Stage } 5^{\dagger} \\
\text { Hemodialysis } \\
\text { Peritoneal dialysis }\end{array}$ & $\begin{array}{l}20 \\
25\end{array}$ & $\begin{array}{l}30 \\
45\end{array}$ & $\begin{array}{l}50 \\
20\end{array}$ & $\begin{array}{l}45 \\
50\end{array}$ \\
\hline
\end{tabular}

Reproduced and modified with permission from Kasiske. ${ }^{20}$

* Data from National Health and Nutrition Examination Survey (NHANES) III and the Framingham Offspring Study. ${ }^{54,55}$

${ }^{\dagger}$ Data extracted from multiple observational studies Kasiske. ${ }^{20}$

${ }^{\ddagger}$ Nephrotic proteinuria was defined as $>3 \mathrm{~g}$ of total protein excretion in 24 hours.

CKD, chronic kidney disease; LDL, low-density lipoprotein; HDL, high-density lipoprotein.

In this review, we will discuss the following issues in patients with CKD: 1) changes in lipid metabolism; 2) prevalence of dyslipidemia; 3) associations between dyslipidemia and all-cause and CVD mortality; 4) data on therapy for dyslipidemia; 5) safety of lipid-lowering therapy; 6) NKF K/DOQI guidelines on treating dyslipidemia; and 7) areas where data are limited and additional research is required.

\section{Overview of Lipid Metabolism in CKD}

Lipoproteins traditionally are classified based on their densities, ranging from chylomicrons to high-density lipoprotein cholesterol (HDL-C), and consist of cholesterol, triglycerides, phospholipids, and proteins. Apolipoproteins comprise the protein component of the lipoproteins and serve as binding ligands for cholesterol receptors. Specific lipoproteins are associated with particular apolipoproteins; for example, LDL-C contains high amounts of apolipoprotein (apo) B.

In $\mathrm{CKD}$, the etiology of dyslipidemia may be reflected more accurately in the apolipoprotein profile. Levels of apoA-I and apoA-II are often reduced, yielding decreased production of HDL-C. In addition, apoC-III, integral for the metabolism of both LDL-C and very low-density lipoprotein cholesterol (VLDL-C), is present in elevated levels. The accumulation of apoB-containing VLDL particles is also important in the development and maintenance of dyslipidemia in CKD. ${ }^{16}$ Factors that may contribute to decreased catabolism and clearance of triglyceride-rich apoB-containing lipoproteins include 1) reduced activity of lipolytic enzymes; 2) compositional abnormalities in lipoproteins preventing binding to appropriate receptors; and 3) decreased uptake of lipoproteins from the circulation. ${ }^{17}$ Binding and uptake of lipoproteins may be affected by increased amounts of oxidant stress seen in CKD such that oxidative modification of lipoproteins leads to decreased uptake by the appropriate receptors and subsequent atherosclerosis. ${ }^{18}$ In summary, multiple factors interfere with uptake of triglyceride- rich, apoB-containing lipoproteins by the liver and in the periphery, yielding increased circulation of these potentially atherogenic lipoproteins. ${ }^{19}$

\section{Prevalence of Dyslipidemia in CKD}

Dyslipidemia is common in patients with $\mathrm{CKD}$, and the lipid profile varies widely depending on the level of kidney function and the degree of proteinuria (Table 1$).{ }^{20}$ Prior to developing $\mathrm{CKD}$, patients frequently have elevated total and LDL-C levels. However, as CKD advances to kidney failure, the prevalence of elevated total and LDL-C levels decreases. In dialysis patients, LDL-C levels generally are lower than in the general population. Still, half of all dialysis patients have either LDL-C levels over $100 \mathrm{mg} / \mathrm{dl}(2.6 \mathrm{mmol} / \mathrm{L})$ or non-HDL-C levels over $130 \mathrm{mg} / \mathrm{dl}(3.4 \mathrm{mmol} / \mathrm{L}) .^{3}$ Furthermore, HDL-C often is low in hemodialysis patients, while triglycerides generally are moderately elevated. Other abnormalities include increased levels of lipoprotein(a), a higher proportion of atherogenic oxidized LDL-C, and abnormalities in the composition of the lipoproteins as discussed earlier. The classic lipid profile of late-stage CKD includes hypertriglyceridemia, low HDL-C, and low or normal LDL-C, a profile similar to that often seen in patients with diabetes and the metabolic syndrome. ${ }^{16}$

In peritoneal dialysis (PD) patients, the prevalence of LDL-C levels greater than $100 \mathrm{mg} / \mathrm{dl}(2.6 \mathrm{mmol} / \mathrm{L})$ is approximately $70 \%,{ }^{3}$ and $45 \%$ of this population have LDLC levels above $130 \mathrm{mg} / \mathrm{dl}$ (3.4 mmol/L) (Table 1). Overall, PD patients have a somewhat more atherogenic lipid panel than hemodialysis patients, with increased LDL-C, apolipoprotein B, oxidized LDL-C, triglycerides, and lipoprotein(a) as well as decreased HDL-C. This may be due to the near-universal use of glucose-containing peritoneal dialysate and subsequent absorption of glucose across the peritoneal membrane. ${ }^{17}$

In the adult kidney transplant population, several studies have also shown that dyslipidemia is highly prevalent. Over $80 \%$ of patients have total cholesterol levels 


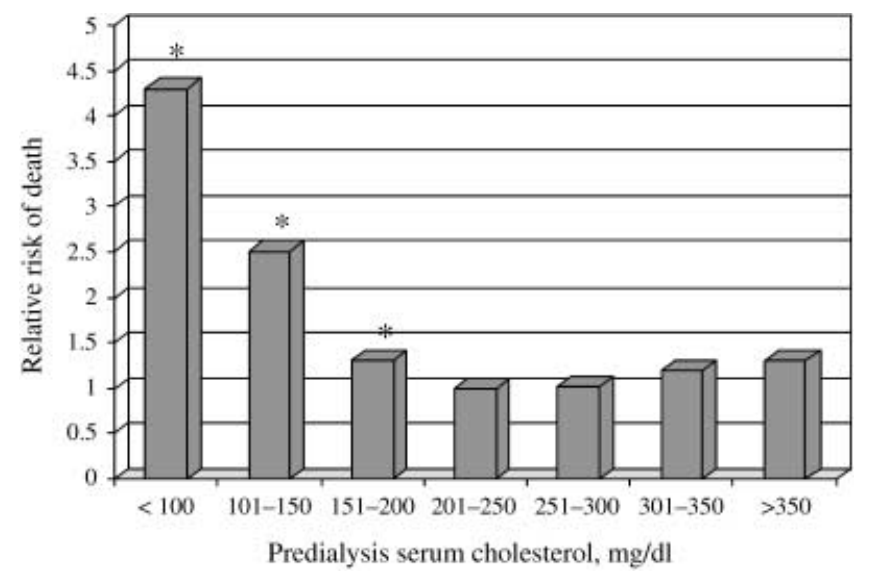

FIGURE 1. Relative risk of death in hemodialysis according to serum total cholesterol concentration. Reference group is 200-250 mg/dl. Reprinted with permission from Lowrie EG and Lew NL. ${ }^{24}{ }^{*} P<.001$.

above $200 \mathrm{mg} / \mathrm{dl}(5.2 \mathrm{mmol} / \mathrm{L})$ and over 90\% have LDL-C levels above $100 \mathrm{mg} / \mathrm{dl}(2.6 \mathrm{mmol} / \mathrm{L}) .{ }^{21-23}$

\section{Relationship Between Serum Cholesterol Levels and Mortality in CKD}

Clinical trials in the general population have demonstrated that coronary heart disease (CHD) mortality reduces proportionally with the rate of LDL-C level reduction. In patients with $\mathrm{CKD}$, the relationship between total cholesterol levels and CHD mortality is more complex. A review of observational studies of dialysis patients has for the most part noted a "reverse epidemiology" between total cholesterol levels and risk of all-cause mortality such that lower cholesterol levels are associated with a higher mortality rate. ${ }^{20}$ For example, in an analysis of data from more than 12,000 hemodialysis patients, patients with low total cholesterol levels (less than $100 \mathrm{mg} / \mathrm{dl}$ [2.6 mmol/L]) had over 4 times the mortality risk of patients with total cholesterol levels between 200 and $250 \mathrm{mg} / \mathrm{dl}$ (5.2-6.5 mmol/L) (Fig. 1). ${ }^{24}$

A more recent 10-year prospective study evaluated the importance of total cholesterol levels in 1,167 chronic hemodialysis patients in Japan. ${ }^{25}$ When compared to a reference group with total cholesterol between 200 and $220 \mathrm{mg} / \mathrm{dl}$ (5.2-5.7 mmol/L), hypocholesterolemia was associated with a significantly higher all-cause mortality rate. Hypocholesterolemia also correlated closely with low serum albumin and high serum C-reactive protein (CRP) levels, perhaps identifying hypocholesterolemia as a surrogate for malnutrition or inflammation. Indeed, in patients with high serum albumin levels, elevated total cholesterol was a strong predictor of cardiovascular death. Similarly, in another study of dialysis patients, in the absence of inflammation and malnutrition, elevated total cholesterol was associated with increased risk of CVD events, while the presence of inflammation attenuated the relationship between hypocholesterolemia and CVD. ${ }^{26}$

\section{Therapy for Dyslipidemia in CKD-Statins}

Three important issues to assess are 1) whether statin therapy effectively and safely reduces dyslipidemia in the CKD population; 2) whether improvement of dyslipidemia in the CKD population is associated with reduced CVD events; and 3) whether statins exert other beneficial effects.

\section{Efficacy of Statin Therapy in Reducing Lipid Levels in Patients with CKD}

Peritoneal Dialysis Patients. Only 3 small trials have evaluated statin therapy in patients undergoing PD. A randomized, placebo-controlled study assessed the effect of atorvastatin in 177 patients undergoing continuous ambulatory peritoneal dialysis (CAPD). ${ }^{27}$ After 16 weeks, patients receiving atorvastatin experienced greater reductions in LDL-C and triglyceride levels and significant increases in HDL-C levels compared with subjects receiving placebo. A 4-month, nonrandomized study evaluating the effect of atorvastatin in 29 hypercholesterolemic patients undergoing PD showed similar results. ${ }^{28}$ Finally, significant reduc-tions in LDL-C levels compared with placebo were noted in a 24-week randomized study of simvastatin $10 \mathrm{mg} /$ day in 23 patients undergoing CAPD. ${ }^{29}$

Hemodialysis Patients. Four studies to date have assessed the effect of statin therapy on LDL-C levels in patients undergoing hemodialysis, and all 4 studies demonstrated that statin therapy reduced LDL-C levels. In one study, 34 hemodialysis patients randomized to therapy with simvastatin experienced a large, statistically significant reduction in LDL-C levels and a smaller but significant reduction in triglyceride levels compared with placebo. ${ }^{29}$ An 8-week long randomized trial in 58 hemodialysis patients also noted that simvastatin significantly reduced LDL-C levels, ${ }^{30}$ while a 24-week, nonrandomized study of simvastatin $5 \mathrm{mg} /$ day demonstrated significant reductions in LDL-C levels in 38 hypercholesterolemic patients. ${ }^{31}$ Finally, a randomized study of atorvastatin and simvastatin found that both statins significantly reduced LDL-C levels. ${ }^{32}$

Kidney Transplant Recipients. The use of statins in kidney transplant patients has been well investigated. In addition to several small studies that have demonstrated a significant reduction in LDL-C levels with statins, ${ }^{33-35}$ the results of a large, randomized, placebo-controlled trial are available. The Assessment of LEscol in Renal Transplantation (ALERT) trial was designed to examine long-term cardiovascular outcomes. ${ }^{36}$ After a mean follow-up of 5.1 years, kidney transplant patients receiving fluvastatin 40 to $80 \mathrm{mg} /$ day ( $N=1,050)$ experienced a $32 \%$ reduction in LDL-C compared with patients receiving placebo $(N=1,052)$. 


\section{Statins and CVD Events}

Stages 2 to 4 CKD. Few studies have examined this question in CKD. The best evidence to date comes from a posthoc subgroup analysis of the Cholesterol and Recurrent Events (CARE) study, focusing on patients with Stages 2$4 \mathrm{CKD}^{37}$ The CARE study was a randomized, doubleblinded, placebo-controlled trial of pravastatin in patients with a history of myocardial infarction and total plasma cholesterol levels below $240 \mathrm{mg} / \mathrm{dl}(6.2 \mathrm{mmol} / \mathrm{L})$. Among 1,711 subjects with estimated creatinine clearance less than $75 \mathrm{ml} / \mathrm{min}$ (mean creatinine clearance, $64 \mathrm{ml} / \mathrm{min}$ ), a $28 \%$ reduction in the composite study outcome of myocardial infarction and fatal CHD for the pravastatin group was noted, but there was no significant difference in allcause mortality. Similar risk reductions associated with pravastatin therapy were noted in all subjects regardless of kidney function as well as in subjects with creatinine clearance less than $60 \mathrm{ml} / \mathrm{min}$.

Stage 5 CKD. No randomized controlled trials to date have assessed whether statin therapy reduces the rate of CVD events in dialysis patients. Limited data include a recent observational study that compared 362 hemodialysis patients receiving statin therapy with 3,354 patients not receiving statins and noted an independent association between statin use and a reduced risk of total mortality and cardiovascular-specific mortality. ${ }^{38}$ The association between statin use and reduced CVD mortality held only for patients with known preexisting CVD, and the conclusions are limited by the observational nature of the study.

Kidney Transplant Recipients. To date, only a single trial (ALERT) has assessed whether there was a reduction in the rate of CVD events in kidney transplant patients with statin therapy. ${ }^{36}$ In this trial, there was a trend toward reduction of the composite endpoint (cardiac death, nonfatal myocardial infarction, or coronary intervention procedure) with fluvastatin as compared to placebo (relative risk [RR], 0.83; 95\% confidence interval [CI], 0.64 to 1.06$)$. There were significantly fewer cardiac deaths and nonfatal myocardial infarctions in the treatment group but no reduction was seen for coronary intervention procedures, cerebrovascular events, noncardiovascular death, all-cause mortality, graft loss, or doubling of serum creatinine levels.

\section{Other Effects of Statins}

Statins may exert their benefits on reductions in CVD in the general population either by lowering cholesterol or through other pleiotropic effects. In this section, we review the 1) anti-inflammatory effects of statins in the general population and in $\mathrm{CKD}$, and 2) the potential for statins to reduce the progression of kidney disease.

Anti-inflammatory Effects. Inflammation increases oxidation in the cardiovascular system by increasing levels of atherogenic oxidized LDL-C. A marker of inflammation, Creactive protein (CRP) has been extensively studied as an indicator of cardiovascular risk. In hemodialysis patients, significant reductions in CRP levels from baseline were observed in one observational study after 8 weeks of simvastatin therapy. ${ }^{30}$ However, despite reductions in LDL-C oxidation, no significant change in CRP levels was noted in another single-blinded, randomized trial of statins in 28 hemodialysis patients. ${ }^{32}$ Although anti-inflammatory benefits have been associated with statin use in the general population $^{39}$ and these small studies in hemodialysis patients suggest that statins may reduce inflammation and oxidative stress in CKD, additional research is still required, focusing in particular on clinical outcomes.

Reducing Progression of CKD. Results from small, randomized trials have demonstrated a potential benefit of statins with regard to slowing decline in GFR. A metaanalysis of 12 trials in subjects with CKD demonstrated that patients receiving lipid-lowering therapy, primarily with statins, experienced significantly lower monthly decline in GFR compared with controls $(0.16 \mathrm{ml} / \mathrm{min} /$ month; $95 \%$ CI, 0.03 to 0.29 ); this study also showed a trend toward a reduction of proteinuria with lipid-lowering therapy, although there was considerable heterogeneity among the included studies. ${ }^{40}$ In a recent post-hoc analysis of a subgroup 690 patients with moderate CKD (estimated GFR $<60 \mathrm{ml} / \mathrm{min}$ per $1.73 \mathrm{~m}^{2}$ ) who were part of a randomized controlled trial, statin therapy slowed the decline in kidney function in persons with moderate to severe kidney disease, especially in those with proteinuria. ${ }^{41}$ In another randomized controlled study, Bianchi et al. evaluated atorvastatin 10 to $40 \mathrm{mg} /$ day versus no treatment in 56 patients with CKD receiving antihypertensive medications. ${ }^{42}$ After 1 year, the rate of proteinuria in the atorvastatin-treated patients decreased significantly compared with baseline $(P<.01)$, while there was no change in proteinuria in the control group. Notably, there was wide interpatient variability in efficacy.

In sum, data from several small, randomized controlled trials indicate that statins effectively reduce LDL-C levels in patients with CKD. Because of the paucity of clinical data, it is currently unclear whether statin therapy is associated with a reduced rate of CVD events and whether this effect is only seen in patients at greatest risk. Although large, controlled, randomized trials are lacking, statins may confer other protective effects, such as slowing the decline in GFR or reducing levels of inflammation.

\section{Other Pharmacologic Therapy in CKD}

Fibrates are indicated when hypertriglyceridemia (serum triglycerides $\geq 500 \mathrm{mg} / \mathrm{dl}$ ) is the primary lipid abnormality (Table 2), and may reduce triglyceride levels by up to $30 \%$ to $50 \% .{ }^{3}$ However, fibrates are excreted by the kidney and may cause myositis, particularly when used in conjunction with statins. Therapy with fibrates may also cause an 
Table 2. The Management of Dyslipidemia in Adults with Chronic Kidney Disease

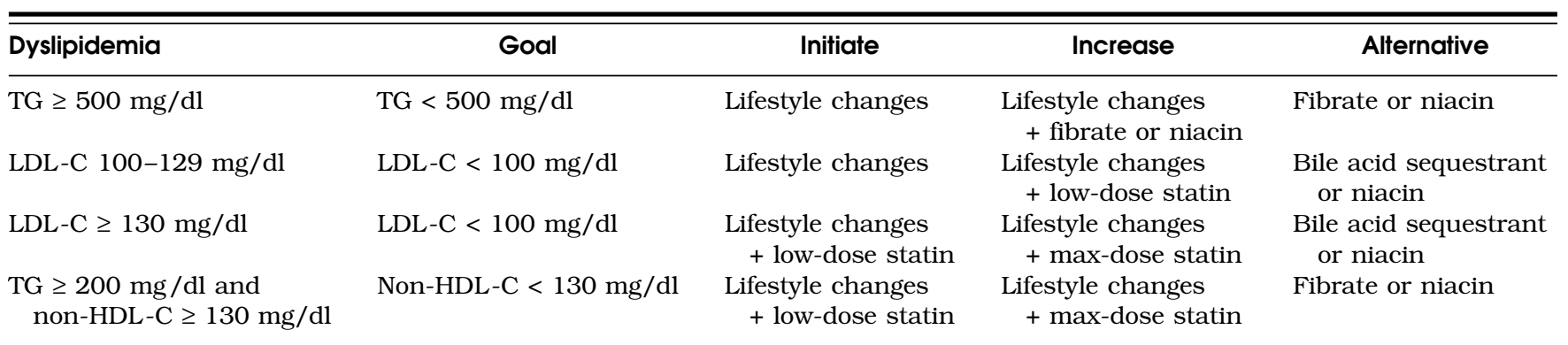

HDL-C, high-density lipoprotein cholesterol; $L D L-C$, low-density lipoprotein cholesterol; TG, triglycerides.

Reproduced with permission from K/DOQI guidelines on treating dyslipidemia. ${ }^{3}$

increase in serum creatinine that is not related to overt muscle injury, although this has not been observed with gemfibrozil. ${ }^{43}$ It is uncertain whether this represents an actual decrease in GFR versus an assay effect, an alteration in creatinine secretion, or an increase in creatinine production. $^{44-46}$

Nicotinic acid (niacin) is the most efficacious agent for increasing HDL-C levels in the general population and also has beneficial effects on triglyceride levels and less significant but favorable LDL-C-lowering effects. ${ }^{47}$ Although the side effect profile of niacin may be more of an issue than with statins, ${ }^{48}$ newer long-acting niacin preparations may improve tolerability. Extended-release preparations of niacin have recently been studied in patients with diabetes mellitus with dyslipidemia profiles similar to that seen in CKD. This study showed significant improvement in HDL-C, LDL-C, and triglyceride levels with a daily dose of $1,500 \mathrm{mg}$, as well as only small, if any, changes in diabetes control. Unfortunately, kidney disease patients were not discussed in this study. ${ }^{49}$ Additional research is needed to demonstrate the safety, efficacy, and appropriate use of niacin in patients with CKD.

Bile acid sequestrants work by binding bile acids in the intestine, blocking distal reabsorption and thereby decreasing LDL-C levels. Bile acid sequestrants are not well studied in patients with CKD, but are probably well tolerated given their lack of systemic absorption. Bile acid sequestrants currently are recommended as second-line agents for reducing elevated LDL-C levels (Table 2).

\section{Safety of Pharmacologic Therapy in CKD}

The treatment of patients with CKD can be quite complex because of the many comorbid conditions in this population. Given the use of multiple medications as well as the changes in metabolism of medications, it is important to remain aware of dose adjustments and potential medication interactions.

Generally, most lipid-lowering therapies are safe and well tolerated. Table 3 presents dosing recommendations and several contraindications for lipid-lowering medications. For statins, the acceptable dosage varies according to the statin used and the level of kidney function. In general, statins that are not metabolized by the kidney are well tolerated at all doses. Indeed, doses of atorvastatin of up to $80 \mathrm{mg} /$ day produced no serious adverse events in hemodialysis patients. ${ }^{50}$ Although statins are first-line therapy for most dyslipidemias, other lipid-lowering medications may be used in dialysis patients with attention paid to dosage and side effects (Table 3).

Before initiating statin therapy in CKD patients, it may be helpful to establish the patient's baseline creatine phosphokinase (CPK) levels, so that if adverse effects such as myositis occur, the patient can be evaluated more readily. If a patient reports muscle pain, statins should be withdrawn and CPK levels assessed. Patients with only mild adverse reactions to their initial statin regimen could be prescribed the same statin at a lower dosage or started on a different statin before proceeding to alternative lipidlowering therapies. ${ }^{3}$

Statins are contraindicated for patients with acute or chronic liver disease and close attention should be paid to interactions that affect statin metabolism. Agents known to increase statin blood levels include calcineurin inhibitors, such as cyclosporine and tacrolimus, which are commonly used in kidney transplant recipients. Other agents that interact with statins include macrolide antibiotics, azole antifungal agents, calcium channel blockers (particularly nondihydropyridines), fibrates, nicotinic acid, serotonin reuptake inhibitors, warfarin, and grapefruit juice. Statin therapy in conjunction with a fibrate can increase the risk of myositis or rhabdomyolysis; K/DOQI guidelines recommend avoiding this combination in patients with $\mathrm{CKD} .^{3}$

\section{Recommendations from the K/DOQI Dyslipidemia Guidelines}

Because of the increased risk of CVD and the high prevalence of dyslipidemia in patients with CKD, the NKF recently released guidelines for the treatment of dyslipidemia in these patients. ${ }^{3}$ In developing these guidelines, the NKF dyslipidemia work group considered a number of issues. First, the benefits of reduced cholesterol levels in the general population can be extrapolated to patients with 
Table 3. Lipid-lowering Medication Dose Adjustments for Reduced Kidney Function

\begin{tabular}{|c|c|c|c|c|}
\hline \multicolumn{5}{|c|}{ GFR $\left(\mathrm{ml} / \mathrm{min} / 1.73 \mathrm{~m}^{2}\right)$} \\
\hline Agent & 60 to 90 & 15 to 59 & $<15$ & Notes \\
\hline Atorvastatin & No & No & No & \\
\hline Fluvastatin & No & $\downarrow$ to $50 \%$ & $\downarrow$ to $50 \%$ & Decrease dosage by half at GFR $<30$ \\
\hline Lovastatin & No & $\downarrow$ to $50 \%$ & $\downarrow$ to $50 \%$ & Decrease dosage by half at GFR $<30$ \\
\hline Simvastatin & ? & $?$ & $?$ & Start at $5 \mathrm{mg} /$ day in patients with GFR $<10$ \\
\hline Nicotinic acid & No & No & $\downarrow$ to $50 \%$ & $\begin{array}{l}\text { May worsen glycemic control and cause orthostasis, } \\
\text { hyperuricemia, and flushing }\end{array}$ \\
\hline \multicolumn{5}{|c|}{ Bile acid sequestrant } \\
\hline Cholestyramine & No & No & No & Not absorbed \\
\hline Clofibrate & $\downarrow$ to $50 \%$ & $\downarrow$ to $25 \%$ & Avoid & May increase serum creatinine $^{\dagger}$ \\
\hline Fenofibrate & $\downarrow$ to $50 \%$ & $\downarrow$ to $25 \%$ & Avoid & May increase serum creatinine ${ }^{\dagger}$ \\
\hline Gemfibrozil & No & No & No & Likely no effect on serum creatinine \\
\hline
\end{tabular}

* Because of increased risk of myositis and rhabdomyolysis, statin therapy in conjunction with a fibrate should be avoided in patients with chronic kidney disease.

${ }^{\dagger}$ The increase in levels of serum creatinine seen with most fibrates has not been appreciated with gemfibrozil.

GFR, glomerular filtration rate in $\mathrm{ml} / \mathrm{min} / 1.73 \mathrm{~m}^{2}$;

Reproduced with permission from NKF K/DOQI guidelines on treating dyslipidemia. ${ }^{3}$

CKD only if dyslipidemia is thought to affect patients with $\mathrm{CKD}$ in a way similar to the general population. Second, the duration of therapy required to improve cholesterol levels, and hence to reduce the rate of CVD, should not exceed the patient's life expectancy. Third, the safety and tolerability of any proposed therapy must be proven.

The work group concluded that much of the National Cholesterol Education Program Adult Treatment Panel III (NCEP ATP III) guidelines ${ }^{5}$ were applicable to patients with
Stages 1-5 CKD, including kidney transplant patients, with several important exceptions. The K/DOQI guidelines for the treatment of dyslipidemia in $\mathrm{CKD}$ are summarized in Table 2. The key features in the NKF K/DOQI recommendations that differ from NCEP ATP III are summarized in Table 4. Most notable among K/DOQI recommendations is that all stages of CKD are considered a CHD risk equivalent (similar to diabetes mellitus); therefore, all patients with CKD should be considered as belonging to the highest

Table 4. Key Features of the K/DOQI Guidelines that Differ from NCEP ATP III

\section{NKF K/DOQI Guidelines}

CKD patients are considered to be in the highest risk category Evaluation of dyslipidemias should occur at presentation, after a change in status, and annually

Drug therapy should be used for LDL 100 to $129 \mathrm{mg} / \mathrm{dl}$ after only 3 months of TLC

Initial drug therapy for elevated LDL should be with a statin

Fibrates may be used in Stage 5 CKD 1) for patients with triglycerides $\geq 500$; and 2) for patients with triglycerides $\geq 200 \mathrm{mg} / \mathrm{dl}$ with non-HDL cholesterol $\geq 130 \mathrm{mg} / \mathrm{dl}$ who do not tolerate statins

Gemfibrozil may be the fibrate of choice for treatment of high triglycerides in patients with CKD

\section{ATP III Guidelines}

CKD patients are not managed differently from other patients Evaluation of dyslipidemias should occur every 5 years

Drug therapy considered optional for LDL $100-129 \mathrm{mg} / \mathrm{dl}$

Initial drug therapy for elevated LDL should be with a statin, bile acid sequestrant, or nicotinic acid

Fibrates are contraindicated in CKD

No preferences for which fibrate should be used for hypertriglyceridemia

CKD, chronic kidney disease; NKF K/DOQI, National Kidney Foundation Kidney Disease Outcomes Quality Initiative; NCEP ATP III, National Cholesterol Education Program Adult Treatment Panel III; LDL, low-density lipoprotein; HDL, high-density lipoprotein; TLC, therapeutic lifestyle changes.

Reproduced and modified with permission from the NKF K/DOQI guidelines on treating dyslipidemia. ${ }^{3}$ 
risk group for CHD and target LDL-C should be less than $100 \mathrm{mg} / \mathrm{dl}(2.6 \mathrm{mmol} / \mathrm{L})$.

The guidelines recognize that some CKD patients with dyslipidemia may be able to achieve their cholesterol goals through lifestyle changes alone. However, because of the complex dietary requirements of these patients, particulary those with stage $5 \mathrm{CKD}$, consultation with a specialist dietician is important. Realistically, most CKD patients with dyslipidemia eventually will require pharmacologic treatment.

\section{Limitations of Existing Data and Ongoing Trials}

It remains unknown whether the reverse epidemiology with regard to cholesterol and mortality that is seen in dialysis patients is due solely to confounding factors (for example, malnutrition) that both decrease cholesterol and increase mortality risk, or whether the complex lipid profile in dialysis patients leads to a substantially different relationship between lipid levels and cardiovascular events. There are three ongoing trials that are evaluating the efficacy of statins in reducing CVD in patients with CKD. The Study of Heart and Renal Protection (SHARP) is a prospective, randomized, placebo-controlled trial that will enroll 6,000 predialysis patients and 3,000 dialysis patients. ${ }^{51}$ Its primary goal is to assess the efficacy of cholesterol-lowering therapy (ezetimibe and simvastatin combined vs placebo) on the time to a first major vascular event. It began in 2003 and has 4 years of follow-up planned. The AURORA study is a prospective, randomized, placebo-controlled trial in 3,000 hemodialysis patients evaluating the effect of rosuvastatin on the rate of cardiovascular events. ${ }^{52}$ AURORA began in 2003 and also has approximately 4 years of followup planned. Finally, the Die Deutsche Diabetes Dialyse (4D) Study is a prospective, randomized, placebo-controlled trial that has enrolled 1,200 hemodialysis patients with type 2 diabetes. ${ }^{53}$ This study will assess the effect of atorvastatin $20 \mathrm{mg} /$ day on the combined incidence of nonfatal myocardial infarction and cardiovascular mortality. This trial is expected to be completed in 2004. Together, these studies will contribute to our knowledge of the optimal treatment of these high-risk patients.

\section{Conclusions}

Patients with CKD present a challenge to the physician both because of the complexity of their condition and because their lipid profiles and treatment requirements evolve as their disease progresses. Although statin therapy appears safe in patients with CKD and is the first-line recommendation for patients with LDL-C levels greater than $100 \mathrm{mg} / \mathrm{dl}(>2.6 \mathrm{mmol} / \mathrm{L})$ and/or triglyceride levels less than $500 \mathrm{mg} / \mathrm{dl}(<5.6 \mathrm{mmol} / \mathrm{L})$, there are currently insufficient data evaluating the efficacy of statin therapy in reducing CVD events in this population. Because CKD patients, and in particular the subset receiving dialysis, are at high risk for coronary events, this lack of data represents a signifi- cant gap in our knowledge base. The results of ongoing randomized trials should provide more definitive data on the risks and benefits of lipid-lowering therapy in this population.

Grant funding for Dr. Sarnak is via NIH grant K23 NIDDK 02904.

Dr. Weiner is funded by NIH training grant T32DK007777.

\section{REFERENCES}

1. Levey AS, Coresh J, Balk E, et al. National Kidney Foundation practice guidelines for chronic kidney disease: evaluation, classification, and stratification. Ann Intern Med. 2003;139:137-47.

2. Prichard S. Risk factors for coronary artery disease in patients with renal failure. Am J Med Sci. 2003;325:209-13.

3. National Kidney Foundation. K/DOQI clinical practice guidelines for managing dyslipidemias in chronic kidney disease. Am J Kidney Dis. 2003;41(suppl 3):S1-S92.

4. National Kidney Foundation. K/DOGI clinical practice guidelines for chronic kidney disease: evaluation, classification, and stratification. Am J Kidney Dis. 2002;39(suppl 1):S1-S237.

5. Executive Summary of the Third Report of the National Cholesterol Education Program (NCEP) Expert Panel on Detection, Evaluation, and Treatment of High Blood Cholesterol in Adults (Adult Treatment Panel III). JAMA. 2001;285:2486-97.

6. Sarnak MJ, Levey AS. Cardiovascular disease and chronic renal disease: a new paradigm. Am J Kidney Dis. 2000;35(4 suppl 1):S1 17-S131.

7. Weiner DE, Tighiouart H, Amin MG, et al. Chronic kidney disease as a risk factor for cardiovascular disease and all-cause mortality: a pooled analysis of community-based studies. J Am Soc Nephrol. 2004; 15:1307-15.

8. Stehouwer CD, Gall MA, Twisk JW, Knudsen E, Emeis JJ, Parving HH. Increased urinary albumin excretion, endothelial dysfunction, and chronic low-grade inflammation in type 2 diabetes: progressive, interrelated, and independently associated with risk of death. Diabetes. 2002;51:1157-65.

9. Clausen P, Jensen JS, Jensen G, Borch-Johnsen K, Feldt-Rasmussen B. Elevated urinary albumin excretion is associated with impaired arterial dilatory capacity in clinically healthy subjects. Circulation. 2001;103:1869-74.

10. Sarnak MJ, Levey AS, Schoolwerth AC, et al. Kidney disease as a risk factor for development of cardiovascular disease: a statement from the American Heart Association Councils on kidney in cardiovascular disease, high blood pressure research, clinical cardiology, and epidemiology and prevention. Circulation. 2003;108:2154-69.

11. McKenney JM. Lipid management: tools for getting to the goal. Am J Manag Care. 2001;7(9 suppl):S299-S306.

12. Prevention of cardiovascular events and death with pravastatin in patients with coronary heart disease and a broad range of initial cholesterol levels. The Long-Term Intervention with Pravastatin in Ischaemic Disease (LIPID) Study Group. N Engl J Med. 1998;339: 1349-57.

13. Sever PS, Dahlof B, Poulter NR, et al. Prevention of coronary and stroke events with atorvastatin in hypertensive patients who have average or lower-than-average cholesterol concentrations, in the Anglo-Scandinavian Cardiac Outcomes Trial-Lipid Lowering Arm (ASCOT-LLA): a multicentre randomised controlled trial. Lancet. 2003;361:1149-58.

14. Randomised trial of cholesterol lowering in 4444 patients with coronary heart disease: the Scandinavian Simvastatin Survival Study (4S). Lancet. 1994;344:1383-9.

15. MRC/BHF Heart Protection Study of cholesterol lowering with simvastatin in 20,536 high-risk individuals: a randomised placebocontrolled trial. Lancet. 2002;360:7-22. 
16. Attman PO, Knight-Gibson C, Tavella M, Samuelsson O, Alaupovic $P$. The compositional abnormalities of lipoproteins in diabetic renal failure. Nephrol Dial. Transplant. 1998;13:2833-41.

17. Attman PO, Samuelsson O, Johansson AC, Moberly JB, Alaupovic P. Dialysis modalities and dyslipidemia. Kidney Int Suppl. 2003;84: S110-S112.

18. Guaschning T, Krane V, Metzger T, Wanner C. Abnormalities in uremic lipoprotein metabolism and its impact on cardiovascular disease. Am J Kidney Dis. 2001;38(4 suppl 1):S14-S19.

19. Attman PO, Samuelsson O, Alaupovic P. Lipoprotein metabolism and renal failure. Am J Kidney Dis. 1993;21:573-92.

20. Kasiske BL. Hyperlipidemia in patients with chronic renal disease. Am J Kidney Dis. 1998;32(5 suppl 3):S142-S156.

21. Aakhus S, Dahl K, Wideroe TE. Hyperlipidaemia in renal transplant patients. J Intern Med. 1996;239:407-15.

22. Gonyea JE, Anderson CF. Weight change and serum lipoproteins in recipients of renal allografts. Mayo Clin Proc. 1992;67:653-7.

23. Moore R, Thomas D, Morgan E, et al. Abnormal lipid and lipoprotein profiles following renal transplantation. Transplant Proc. 1993; 25(1 pt 2):1060-1.

24. Lowrie EG, Lew NL. Death risk in hemodialysis patients: the predictive value of commonly measured variables and an evaluation of death rate differences between facilities. Am $\mathrm{J}$ Kidney Dis. 1990; 15:458-82.

25. Iseki K, Yamazato M, Tozawa M, Takishita S. Hypocholesterolemia is a significant predictor of death in a cohort of chronic hemodialysis patients. Kidney Int. 2002;61:1887-93.

26. Liu Y, Coresh J, Eustace JA, et al. Association between cholesterol level and mortality in dialysis patients: role of inflammation and malnutrition. JAMA. 2004;291:451-9.

27. Harris KP, Wheeler DC, Chong CC. A placebo-controlled trial examining atorvastatin in dyslipidemic patients undergoing CAPD. Kidney Int. 2002;61:1469-74.

28. Hufnagel G, Michel C, Vrtovsnik F, Queffeulou G, Kossari N, Mignon F. Effects of atorvastatin on dyslipidaemia in uraemic patients on peritoneal dialysis. Nephrol Dial Transplant. 2000;15:684-8.

29. Saltissi D, Morgan C, Rigby RJ, Westhuyzen J. Safety and efficacy of simvastatin in hypercholesterolemic patients undergoing chronic renal dialysis. Am J Kidney Dis. 2002;39:283-90.

30. Chang JW, Yang WS, Min WK, Lee SK, Park JS, Kim SB. Effects of simvastatin on high-sensitivity C-reactive protein and serum albumin in hemodialysis patients. Am J Kidney Dis. 2002;39:1213-7.

31. Nishikawa O, Mune M, Miyano M, et al. Effect of simvastatin on the lipid profile of hemodialysis patients. Kidney Int Suppl. 1999;71:S219-S221.

32. van den Akker JM, Bredie SJ, Diepenveen SH, van Tits LJ, Stalenhoef $\mathrm{AF}$, van Leusen $\mathrm{R}$. Atorvastatin and simvastatin in patients on hemodialysis: effects on lipoproteins, C-reactive protein and in vivo oxidized LDL. J Nephrol. 2003;16:238-44.

33. Holdaas H, Jardine AG, Wheeler DC, et al. Effect of fluvastatin on acute renal allograft rejection: a randomized multicenter trial. Kidney Int. 2001;60:1990-7.

34. Kasiske BL, Heim-Duthoy KL, Singer GG, Watschinger B, Germain MJ, Bastani B. The effects of lipid-lowering agents on acute renal allograft rejection. Transplantation. 2001;72:223-7.

35. Sahu K, Sharma R, Gupta A, et al. Effect of lovastatin, an HMG CoA reductase inhibitor, on acute renal allograft rejection. Clin Transplant. 2001;15:173-5.

36. Holdaas H, Fellstrom B, Jardine AG, et al. Effect of fluvastatin on cardiac outcomes in renal transplant recipients: a multicentre, randomised, placebo-controlled trial. Lancet. 2003;361:2024-31.
37. Tonelli M, Moye L, Sacks FM, Kiberd B, Curhan G. Pravastatin for secondary prevention of cardiovascular events in persons with mild chronic renal insufficiency. Ann Intern Med. 2003;138:98-104.

38. Seliger SL, Weiss NS, Gillen DL, et al. HMG-CoA reductase inhibitors are associated with reduced mortality in ESRD patients. Kidney Int. 2002;61:297-304.

39. Balk EM, Lau J, Goudas LC, et al. Effects of statins on nonlipid serum markers associated with cardiovascular disease: a systematic review. Ann Intern Med. 2003;139:670-82.

40. Fried LF, Orchard TJ, Kasiske BL. Effect of lipid reduction on the progression of renal disease: a meta-analysis. Kidney Int. 2001;59: 260-9.

41. Tonelli M, Moye L, Sacks FM, Cole T, Curhan GC. Effect of pravastatin on loss of renal function in people with moderate chronic renal insufficiency and cardiovascular disease. J Am Soc Nephrol. 2003;14:1605-13.

42. Bianchi S, Bigazzi R, Caiazza A, Campese VM. A controlled, prospective study of the effects of atorvastatin on proteinuria and progression of kidney disease. Am J Kidney Dis. 2003;41:565-70.

43. Broeders N, Knoop C, Antoine M, Tielemans C, Abramowicz D. Fibrate-induced increase in blood urea and creatinine: is gemfibrozil the only innocuous agent? Nephrol Dial Transplant. 2000; 15: 1993-9.

44. Dierkes J, Westphal S, Luley C. Serum homocysteine increases after therapy with fenofibrate or bezafibrate. Lancet. 1999;354:21920.

45. Hottelart C, El Esper N, Rose F, Achard JM, Fournier A. Fenofibrate increases creatininemia by increasing metabolic production of creatinine. Nephron. 2002;92:536-41.

46. Westphal S, Dierkes J, Luley C. Effects of fenofibrate and gemfibrozil on plasma homocysteine. Lancet. 2001;358:39-40.

47. Miller M. Niacin as a component of combination therapy for dyslipidemia. Mayo Clin Proc. 2003;78:735-42.

48. Hiatt JG, Shamsie SG, Schectman G. Discontinuation rates of cholesterol-lowering medications: implications for primary care. Am J Manag Care. 1999;5:437-44.

49. Grundy SM, Vega GL, McGovern ME, et al. Efficacy, safety, and tolerability of once-daily niacin for the treatment of dyslipidemia associated with type 2 diabetes: results of the assessment of diabetes control and evaluation of the efficacy of niaspan trial. Arch Intern Med. 2002;162:1568-76.

50. Lins RL, Matthys KE, Verpooten GA, et al. Pharmacokinetics of atorvastatin and its metabolites after single and multiple dosing in hypercholesterolaemic haemodialysis patients. Nephrol Dial Transplant. 2003;18:967-76.

51. Baigent C, Landry M. Study of Heart and Renal Protection (SHARP). Kidney Int Suppl. 2003;84:S207-S210.

52. Fellstrom BC, Holdaas H, Jardine AG. Why do we need a statin trial in hemodialysis patients? Kidney Int Suppl. 2003;84:S204S206.

53. Wanner C, Krane V, Ruf G, Marz W, Ritz E. Rationale and design of a trial improving outcome of type 2 diabetics on hemodialysis. Die Deutsche Diabetes Dialyse Studie Investigators. Kidney Int Suppl. 1999;71:S222-S226.

54. Report of the National Cholesterol Education Program Expert Panel on Detection, Evaluation, and Treatment of High Blood Cholesterol in Adults. The Expert Panel. Arch Intern Med. 1988;148:36-69.

55. Schaefer EJ, Lamon-Fava S, Cohn SD, et al. Effects of age, gender, and menopausal status on plasma low density lipoprotein cholesterol and apolipoprotein B levels in the Framingham Offspring Study. J Lipid Res. 1994;35:779-92. 